1 Fundação Oswaldo Cruz (Fiocruz) - Eusébio (CE), Brasil.

Orcid: https://orcid org/0000-0001-84473654;

ivana.barreto@fiocruz.br

2 Fundação Oswaldo Cruz (Fiocruz) - Eusébio (CE), Brasil.

Orcid: https://orcid

org/0000-0003-3676-

9607

vanira.pessoa@fiocruz.br

3 Universidade Regional do Cariri (Urca) - Crato (CE), Brasil.

Orcid: https://orcid. org/0000-0002- 25418441

fatimaantero@uol.com.br

4 Fundação Oswaldo Cruz (Fiocruz) - Eusébio (CE),

Brasil.

Orcid: https://orcid

org/0000-0002-4763-

6773

sharmenia.nuto@fiocruz.br

${ }^{5}$ Fundação Oswaldo Cruz (Fiocruz) - Eusébio (CE), Brasil.

Orcid: https://orcid. org/0000-0001-92951177

robertowiff@gmail.com

6 Universidade Federal do Ceará (UFC) - Fortaleza

(CE), Brasil.

Orcid: https://orcid.

org/0000-0003-0870-

1971

kelengomesr@yahoo.com.br

7 Fundação Oswaldo Cruz (Fiocruz) - Eusébio (CE), Brasil.

Orcid: https://orcid. org/0000-0003-42378995

anyavieira10@gmail.com

${ }^{8}$ Fundação Oswaldo Cruz (Fiocruz) - Eusébio (CE), Brasil.

Orcid: https://orcid. org/0000-0002-33350619

odorico0811@gmail.com

\section{Complexidade e potencialidade do trabalho dos Agentes Comunitários de Saúde no Brasil contemporâneo}

\author{
Complexity and potentiality of the Community Health Workers' labor \\ in contemporary Brazil
}

Ivana Cristina de Holanda Cunha Barreto', Vanira Matos Pessoa², Maria de Fátima Antero de Sousa ${ }^{3}$, Sharmenia de Araújo Soares Nuto ${ }^{\mathbf{4}}$, Roberto Wagner Junior Freire de Freitas ${ }^{\mathbf{5}}$, Kelen Gomes Ribeiro6, Anya Pimental Gomes Fernandes Vieira-Meyer ${ }^{\mathbf{7}}$, Luiz Odorico Monteiro de Andrade 8

DOI: 10.1590/0103-11042018S108

RESUMO O objetivo deste artigo foi analisar o escopo de práticas dos Agentes Comunitários de Saúde (ACS) relacionando-o à situação social e de saúde, bem como os elementos facilitadores e os limitantes. Trata-se de um estudo transversal de abordagem mista, e estratégia explanatória sequencial, realizado em quatro municípios do Ceará. No estudo quantitativo, a amostra de 160 ACS foi aleatória com instrumento estruturado. No qualitativo, realizou-se seis grupos focais e entrevistas. Prevaleceram, na amostra, mulheres (139; 86,9\%), casadas (111; 69,4\%), com renda familiar maior ou igual a 2 salários mínimos (102; 63,7\%), nível técnico incompleto $(68 ; 42,5 \%)$, da zona urbana (114; 71,3\%), atuando como ACS há menos de 10 anos (93; $58,2 \%)$. As principais atividades foram visitação domiciliar de grupos prioritários e cadastramento de famílias. Evidenciou-se a complexidade do trabalho, que inclui ações de promoção e vigilância à saúde como pré-natal, imunizações, hipertensão, diabetes, cuidado com idosos, entre outros. Como limitantes das práticas, identificaram-se: deficiência da formação técnica, suporte reduzido no trabalho e violência. Como potencializadores: educação permanente e gestão participativa. O escopo de práticas dos ACS é complexo e abrangente, incluindo a articulação de políticas públicas no território, o que se constitui em uma potencialidade para promoção da saúde de comunidades vulneráveis.

PALAVRAS-CHAVE Agentes Comunitários de Saúde. Atenção Primária à Saúde. Estratégia Saúde da Família. Sistemas de saúde.

ABSTRACT The aim of this article was to analyze the Community Health Worker' $(\mathrm{CHW})$ scope of practices, relating it to the social and health situation faced, as well as with facilitating and limiting elements of their practice. This is a cross-sectional study utilizing a mixed approach, including sequential explanatory strategy, carried out in four municipalities of Ceará. In the quantitative study, the sample of $160 \mathrm{CHW}$ was randomized, and a structured instrument was used to collect data. In the qualitative study, six focal groups and interviews were carried out. We mainly found women $(139 ; 86.9 \%)$, married $(111 ; 69.4 \%)$, with a family income greater than or equal to 2 minumm wages (102; 63.7\%), incomplete professional trainning $(68 ; 42.5 \%)$, from the urban area 
(114, 71.3\%), acting as $\mathrm{CHW}$ for less than 10 years (93, 58.2\%). The main activities were home visitation of priority groups and families registration. The complexity of the work was evidenced, which includes actions of health promotion and surveillance, such as prenatal care, immunizations, hypertension, diabetes, care for the elderly, among others. As limitations of their practices, we identified: deficiency of technical/professional training, reduced work support, and violence. As facilitators, we observed: permanent education and participatory management practices. The scope of $\mathrm{CHW}$ practices is complex and broad, including the articulation of public policies in the territory, which constitutes a potential for health promotion of vulnerable communities.

KEYWORDS Community Health Workers. Primary Health Care. Family Health Strategy. Health systems.

\section{Introdução}

Em 1987, nove anos após a Declaração de Alma Ata' ${ }^{\mathbf{1}}$, iniciou-se a experiência de institucionalização do Programa de Agentes Comunitários de Saúde (Pacs) no Ceará como uma estratégia abrangente de Atenção Primária à Saúde (APS)2 . O Ceará é um Estado brasileiro com $90 \%$ de seu território situado no semiárido nordestino, caracterizado por ter $17,8 \%^{3}$ da população abaixo da linha de pobreza e grande desigualdade social, com Índice de Gini de 0,62, em 20104. Os Agentes Comunitários de Saúde (ACS) iniciaram suas atividades nas regiões mais pobres do Ceará. Eles eram coordenados por enfermeiros, viviam nas comunidades onde exerciam as atividades e visitavam todas as casas de sua área de atuação, uma vez por mês, para realizar um pequeno número de atividades prioritárias de saúde, direcionadas, principalmente, às crianças menores de dois anos e às gestantes ${ }^{2,5}$.

Após a implantação do Pacs, houve rápida queda na mortalidade infantil no Ceará, cuja taxa reduziu de 60 por mil nascidos vivos, em 1981, para 25 por mil, em 1990, além do incremento da imunização. Na década de
1990, o Pacs foi adotado como 'modelo' pelo Ministério da Saúde (MS), sendo expandido para várias outras regiões do País ${ }^{2,5}$. Em 1993, o programa recebeu o Prêmio Maurice Pate do Fundo das Nações Unidas para a Infância pela contribuição ao bem-estar das crianças $^{2,5}$. No ano seguinte, o MS instituiu o Programa de Saúde da Família, incentivando financeiramente as gestões municipais ${ }^{6}$ para implantação de equipes com um médico, enfermeiro e auxiliar de enfermagem, a fim de trabalharem articuladas aos ACS em um território definido. Nesse período, o escopo de práticas realizadas pelos ACS foi alargado. Ainda nos anos 1990, a Estratégia Saúde da Família (ESF) foi definida como estruturante do Sistema Único de Saúde (SUS), e estendida para todo o País ${ }^{7}$, e, em 2011, por meio do Decreto Federal $n^{0} 7.508$, como a principal porta de entrada do SUS ${ }^{8}$.

Em 2006 foi aprovada a Emenda Constitucional 51 que determinou a admissão de ACS por meio de processo seletivo público e promulgada a Lei $\mathrm{n}^{0} 11.350$ que criou a profissão de ACS, estabelecendo como suas atribuições a prevenção de doenças e a promoção da saúde, mediante ações domiciliares ou comunitárias? 
Passados 30 anos da institucionalização do Pacs, a ESF, no Brasil, abrange 48.605 equipes e 270.867 ACS. No Ceará, tem-se 2.426 equipes e $14.776 \mathrm{ACS}^{10}$. A ESF está presente em 97,5\% dos municípios brasileiros e atende cerca de 146 milhões de pessoas, correspondendo a $70,2 \%$ da população"11.

Concomitantemente à expansão da ESF, houve aceleração do processo de urbanização do País e o aumento da complexidade do quadro epidemiológico, caracterizado na atualidade por uma superposição de agendas ${ }^{12}$, que incluem doenças transmissíveis, como as arborviroses ${ }^{13,14}$, não transmissíveis (doenças cardiovasculares, câncer, agravos em saúde mental), representando hoje a maior carga de doença ${ }^{15}$, além da intensificação das causas externas, registrada nas Américas desde a década de $1980^{16}$, e composta, sobretudo, por homicídios e acidentes de trânsito. Em algumas cidades do Nordeste, como Fortaleza, os homicídios tornaram-se a principal causa de morte na década de $2010^{17}$.

Diversos outros problemas sociais e de saúde foram sendo adicionados às responsabilidades dos ACS, como encaminhar crianças fora da escola para a educação $0^{\mathbf{1 8}}$, acompanhar pessoas com doenças crônicas, acamados, prevenir infestação dos domicílios pelo Aedes aegypti, entre outros. Enquanto isto, os investimentos no processo de educação permanente em saúde para os ACS foram poucos, mesmo com o indicativo de que todos devessem ter uma formação técnica mínima.

O Curso Técnico para ACS (CTACS) foi aprovado pelos Ministérios da Saúde e da Educação, em 2004, tendo um total de 1.800 horas, sendo composto por três Etapas Formativas7. Entretanto, 97,1\% dos ACS do Ceará realizaram, apenas, a Etapa Formativa I.

Além do sucesso da experiência com ACS no Brasil, estudos realizados em diversos países conseguiram demonstrar a eficácia destes no controle de doenças não transmissíveis $^{19}$ e doenças transmissíveis ${ }^{20}$, evidenciando aumento do conhecimento dos pacientes quanto aos cuidados necessários para o controle desses agravos. Mesmo diante de tantas evidências nacionais e internacionais da efetividade do trabalho dos ACS na APS, em 2017, o MS, com a concordância da Comissão Intergestores Tripartite, aprovou uma nova Política de Atenção Básica à Saúde em que a presença do agente comunitário de saúde na equipe de atenção básica deixa de ser obrigatória, colocando em xeque a ESF ${ }^{21}$. Outra medida que provocou fortes questionamentos dos sanitaristas foi a instituição de um programa bilionário para oferta de curso de técnico de enfermagem para todos os ACS no Brasil, o denominado Programa de Formação Técnica para Agentes de Saúde (Profags) ${ }^{22}$. Esta última medida contradiz os princípios e elementos da ESF, caracterizada como rede de APS baseada em equipes multiprofissionais, em que o papel do ACS é fundamentalmente o de promoção da saúde, e, portanto, com necessidade do desenvolvimento de competências específicas para educação e mobilização comunitárias, vigilância à saúde no território, entre outras, o que não está previsto em um curso técnico de enfermagem.

A análise do escopo de práticas dos ACS no Ceará ganha importância na medida em que a experiência brasileira começa a ser aplicada em outros países do mundo, como Angola África ${ }^{23}$, e, por outro lado, é colocada em risco no Brasil pela Política Nacional de Atenção Básica (PNAB) 2017. Dessa forma, compreendendo que o quadro social e de saúde do Ceará tem semelhanças ao de outros estados brasileiros e de países da América Latina e da África, propusemo-nos a analisar o escopo de práticas dos ACS, com relação à situação social e de saúde do Ceará, bem como os elementos facilitadores e os limitantes desta prática.

\section{Material e métodos}

Trata-se de um estudo descritivo, analítico, com delineamento transversal e abordagem mista, utilizando uma estratégia explanatória sequencial ${ }^{24,25}$. Consiste de um recorte da 
pesquisa 'Campo e escopo de práticas profissionais e acesso ao cuidado na ESF no Ceará' que investiga práticas profissionais de médicos, enfermeiros, dentistas e ACS.
Os locais selecionados para o estudo representam realidades municipais distintas, com portes populacionais e localização geográfica variados.

Figura 1. Mapa do estado do Ceará com destaque para os municípios de Fortaleza, Tauá, Eusébio e Cruz

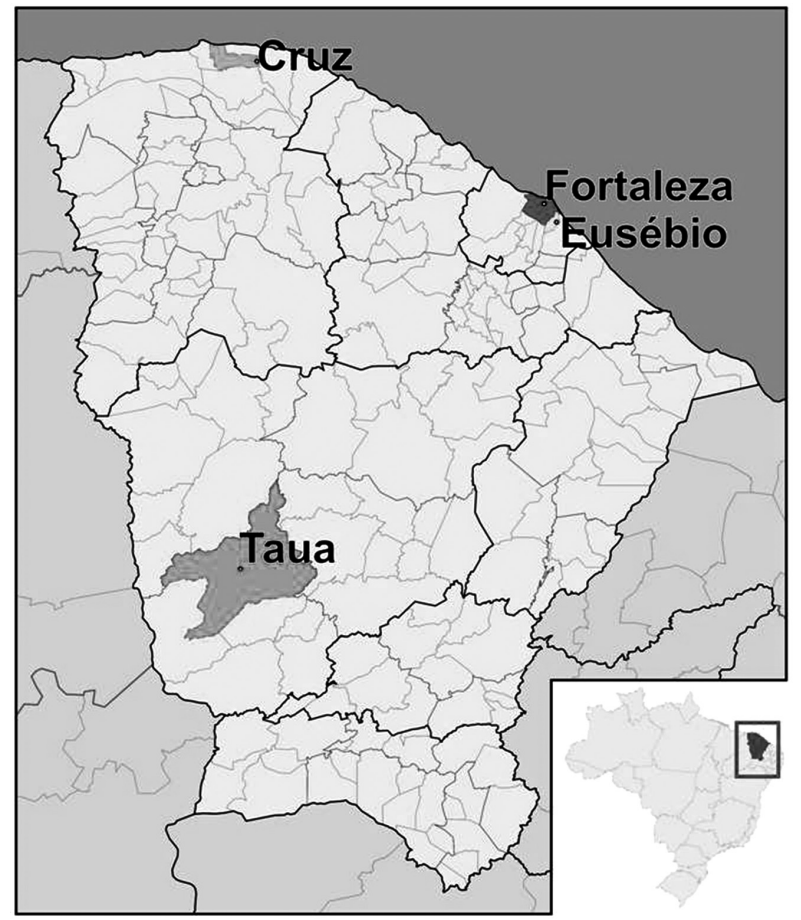

Fonte: Ipece' 26

Quadro 1. Características gerais dos municípios de Fortaleza, Tauá, Eusébio e Cruz - Ceará, 2016

\begin{tabular}{|c|c|c|c|c|}
\hline & Fortaleza & Tauá & Eusébio & Cruz \\
\hline População & 2.571 .896 & 57.478 & 50.308 & 23.514 \\
\hline Densidade Populacional hab. $/ \mathrm{km}^{2}$ & 8.166 & 14 & 636 & 71 \\
\hline População Rural & NA & $42 \%$ & NA & $57 \%$ \\
\hline Cobertura Populacional do PSF & $43 \%$ & $97 \%$ & $95 \%$ & $97 \%$ \\
\hline Número de ESF implantadas & 365 & 28 & 18 & 10 \\
\hline $\begin{array}{l}\text { Percentual de famílias beneficiárias do Bolsa Família } \\
\text { acompanhadas pela ESF }\end{array}$ & 34,35 & 60,72 & 67,00 & 84,4 \\
\hline Rendimento Médio Mensal per capita dos domicílios & 994,24 & 354,53 & 68,16 & 369,18 \\
\hline Localização/Relevo & $\begin{array}{l}\text { Nordeste / Planície } \\
\text { Litorânea }\end{array}$ & $\begin{array}{l}\text { Sudoeste / Depressão } \\
\text { Sertaneja }\end{array}$ & $\begin{array}{l}\text { Nordeste / Tabuleiros } \\
\text { Pré-Litorâneos }\end{array}$ & $\begin{array}{l}\text { Norte / Planície } \\
\text { Litorânea }\end{array}$ \\
\hline
\end{tabular}

Fonte: Ipece'26 
A cidade de Fortaleza, capital do Ceará, encontra-se administrativamente dividida em sete Secretarias Regionais (SR), tendo a pesquisa sido realizada na SR II (363.406 habitantes).

\section{Estudo quantitativo}

A população da pesquisa constituiu-se do total de ACS dos quatro municípios, correspondendo a 256 profissionais. Para o cálculo amostral, foi utilizada fórmula para populações finitas, além de: Intervalo de Confiança (IC) de 95\%, P=50\%, Q=50\% e erro amostral de $4 \%$. A amostra foi aleatória e estratificada por município, totalizando 160 ACS, sendo Cruz - 18, Eusébio - 19, Fortaleza (SER II) - 76, e Tauá - 48 ACS.

Adotaram-se como critérios de inclusão: ser ACS e atuar na função. Foram excluídos aqueles que estavam afastados no momento da coleta dos dados.

A coleta dos dados foi realizada por estudantes e profissionais de saúde, previamente capacitados, sendo as entrevistas realizadas em agosto e setembro/2016. O questionário continha questões estruturadas relativas à: caracterização sociodemográfica, itinerário formativo e práticas profissionais realizadas nos territórios.

As informações, para armazenamento, foram inseridas no EPI-DATA, versão 3.1 e analisadas no software SPSS, versão 23.0 (IBM, SPSS Statistical Database). Foram realizadas análises univariadas e bivariadas. Nas análises bivariadas, as variáveis independentes foram zona de atuação (urbana ou rural) e tempo de serviço como ACS ( $<10$ anos e $>=10$ anos). As variáveis dependentes foram as práticas de trabalho realizadas pelos ACS.

\section{Estudo qualitativo}

Para o estudo qualitativo, foram selecionadas duas equipes com, no mínimo, seis ACS, exceto em Eusébio, onde só havia equipes com no máximo quatro ACS. Outros critérios para seleção das equipes foram estar completas, mínimo de seis meses de funcionamento, infraestrutura e insumos adequados. De outubro a dezembro de 2017, foram realizados 2 Grupos Focais (GF) em Fortaleza, Tauá e Cruz, e 6 entrevistas abertas com ACS de Eusébio, totalizando 45 participantes. Em Tauá e Cruz, foram realizados um GF na Zona Urbana (ZU) e outro na Zona Rural (ZR). Os GF foram gravados, transcritos e analisados por meio de análise de conteúdo temática ${ }^{27}$. Neles, abordamos aspectos referentes às atividades dos ACS, tais como: grupos prioritários, visitas domiciliares, grupos de educação em saúde, e educação permanente.

A pesquisa foi submetida à Plataforma Brasil, tendo sido analisada e obtido autorização do Comitê de Ética em Pesquisa da Fundação Oswaldo Cruz (Fiocruz), por meio do Parecer de número 1.159.936 de 14/07/2015.

\section{Resultados do estudo quantitativo}

A média de idade de ACS foi de 41,4 anos, com desvio padrão de 9,5, idade mínima de 19 e máxima de 65 anos. Prevaleceu na amostra o sexo feminino, pessoas casadas, católicas, com renda familiar igual ou superior a 2 salários mínimos, com nível técnico. A maioria trabalha na ZU (114; 71,3\%) há menos de 10 anos $(93 ; 58,2 \%)$. Em se tratando do vínculo trabalhista, prevaleceu como agente contratante o próprio município; e como vínculo empregatício, servidor público estatutário (tabela 1). 
Tabela 1. Características sociodemográficas e vínculo trabalhista dos ACS por zona de residência e tempo de serviço, Fortaleza, Tauá, Eusébio e Cruz Ceará, 2016

\begin{tabular}{|c|c|c|c|c|c|c|c|}
\hline & Total de ACS & Zona urbana & Zona rural & \multirow{2}{*}{ Valor $p$} & $<10$ anos & $>10$ anos & \multirow[t]{2}{*}{ Valor $p$} \\
\hline & $\mathrm{N}(\%)$ & $\mathrm{N}(\%)$ & $N(\%)$ & & $N(\%)$ & $N(\%)$ & \\
\hline \multicolumn{8}{|l|}{ Sexo } \\
\hline Masculino & $21(13,1)$ & $16(14,0)$ & $5(10,9)$ & $0,591^{1}$ & $16(17,2)$ & $5(7,5)$ & $0,072^{1}$ \\
\hline Feminino & $139(86,9)$ & $98(86,0)$ & $41(89,1)$ & & $77(82,8)$ & $62(92,5)$ & \\
\hline \multicolumn{8}{|l|}{ Estado civil } \\
\hline Solteiro & $49(30,6)$ & $41(36,0)$ & $8(17,4)$ & $0,021^{1}$ & $30(32,3)$ & $19(28,4)$ & $0,598^{1}$ \\
\hline Casados e outros & $111(69,4)$ & $73(64,0)$ & $38(82,6)$ & & $63(67,7)$ & $48(71,6)$ & \\
\hline \multicolumn{8}{|l|}{ Religião } \\
\hline Católica & $99(61,9)$ & $66(57,9)$ & $33(71,7)$ & $0,103^{1}$ & $51(54,8)$ & $48(71,6)$ & $0,031^{1}$ \\
\hline Outras & $74(38,1)$ & $48(42,1)$ & $13(28,3)$ & & $42(45,2)$ & $19(28,4)$ & \\
\hline \multicolumn{8}{|l|}{ Renda familiar } \\
\hline $1 \mathrm{SM}$ & $58(36,3)$ & $29(25,4)$ & $29(63,0)$ & $<0,001^{1}$ & $36(38,7)$ & $22(32,8)$ & $0,446^{1}$ \\
\hline$\geq 2 \mathrm{SM}$ & $102(63,7)$ & $85(74,6)$ & $17(37,0)$ & & $57(61,3)$ & $45(67,2)$ & \\
\hline \multicolumn{8}{|l|}{ Agente contratante } \\
\hline Estado & $39(24,4)$ & $18(15,8)$ & $21(45,7)$ & $<0,001^{1}$ & $4(4,3)$ & $35(52,2)$ & $<0,001$ \\
\hline Município & $121(75,6)$ & $96(84,2)$ & $25(54,3)$ & & $89(95,7)$ & $32(47,8)$ & \\
\hline \multicolumn{8}{|l|}{ Vínculo empregatício } \\
\hline Servidor público estatutário & $150(93,8)$ & $113(99,1)$ & $37(80,4)$ & $<0,001^{1}$ & $84(90,3)$ & $66(98,5)$ & 0,035 \\
\hline Contrato temporário & $10(6,2)$ & $1(0,9)$ & $9(19,6)$ & & $9(9,7)$ & $1(1,5)$ & \\
\hline \multicolumn{8}{|l|}{ Escolaridade } \\
\hline Ensino fundamental & $2(1,3)$ & $2(1,8)$ & NA & $0,194^{2}$ & $2(2,2)$ & NA & $0,004^{2}$ \\
\hline Ensino médio & $38(23,8)$ & $25(21,9)$ & $13(28,3)$ & & $20(21,5)$ & $18(26,9)$ & \\
\hline Ensino técnico & $68(42,5)$ & $44(38,6)$ & $24(52,2)$ & & $31(33,3)$ & $37(55,2)$ & \\
\hline Superior & $42(26,3)$ & $34(29,8)$ & $8(17,4)$ & & $33(35,5)$ & $9(13,4)$ & \\
\hline Especialização & $10(6,3)$ & $9(7,9)$ & $1(2,2)$ & & $7(7,5)$ & $3(4,5)$ & \\
\hline \multicolumn{8}{|l|}{ Formação técnica em ACS } \\
\hline $\operatorname{Sim}$ & $99(61,9)$ & $68(59,6)$ & $31(67,4)$ & 0,361 & $51(54,8)$ & $48(71,6)$ & 0,031 \\
\hline Não & $61(38,1)$ & $46(40,4)$ & $15(32,6)$ & & $42(45,2)$ & $19(28,4)$ & \\
\hline
\end{tabular}

${ }_{1}^{1}$ Teste Qui-Quadrado; 2 Teste Exato de Fisher.

Foi aplicada uma pergunta não estimulada sobre quais eram as ações que eles consideravam como de sua responsabilidade. Cerca de 97\% afirmou visitação domiciliar de grupos prioritários (gestantes, crianças menores de dois anos, idosos, pessoas com diabetes, tuberculose, hanseníase ou acamadas); e $69,4 \%$, o cadastramento de domicílios e pessoas nos Sistemas de Informação em Saúde (e-SUS Atenção Básica - e-SUS $\mathrm{AB}$ ou Sistema de Informação da Atenção Básica - Siab) (tabela 2). A seguir, aparecem ações mais diretamente relacionadas com a prevenção, como a busca ativa para 
vacinas (38,8\%), visitas sobre a prevenção de doenças $(35,0 \%)$, palestras de educação em saúde (27,5\%). A organização de Grupos Terapêuticos (GT), atividade de promoção da saúde que exige sistematização e trabalho em equipe, foi relatada por apenas $13,1 \%$. Os ACS que não participavam da organização de
GT justificaram que sua equipe não desenvolvia esta atividade.

Os ACS com menos de 10 anos de serviço relataram buscas ativas de pessoas e digitação dos cadastros de famílias no e-SUS AB com maior frequência que os com mais de 10 anos de trabalho (tabela 2).

Tabela 2 . Distribuição de ações realizadas pelos ACS por zona de localização da microárea e tempo de serviço, Regional II de Fortaleza, e municípios de Tauá, Eusébio e Cruz - Ceará, 2016

\begin{tabular}{|c|c|c|c|c|c|c|c|}
\hline & \multirow{2}{*}{$\begin{array}{r}\text { Total } \\
\mathrm{N}(\%)\end{array}$} & \multicolumn{2}{|c|}{ Localização da Microárea } & \multirow[t]{2}{*}{ Valor $\mathrm{p}$} & \multicolumn{2}{|c|}{ Tempo de Serviço dos ACS } & \multirow[t]{2}{*}{ Valor $\mathrm{p}$} \\
\hline & & Urbana $n=114$ & Rural $n=46$ & & $<10$ anos & $>10$ anos & \\
\hline \multicolumn{8}{|l|}{$\begin{array}{l}\text { Ações/atividades consideradas de responsabilidade } \\
\text { dos ACS na equipe de saúde da família }\end{array}$} \\
\hline Cadastro dos domicílios no e-SUS-AB ou Siab & $111(69,4)$ & $76(66,7)$ & $35(76,1)$ & $0,242^{1}$ & $61(65,6)$ & $50(74,6)$ & $0,221^{1}$ \\
\hline Digitação dos cadastros no e-SUS-AB ou Siab & $25(15,6)$ & $25(21,9)$ & NA & $0,001^{1}$ & $19(20,4)$ & $6(9,0)$ & $0,049^{1}$ \\
\hline $\begin{array}{l}\text { Busca ativa de pessoas para vacinação, consultas, } \\
\text { exames e outros }\end{array}$ & $62(38,8)$ & $47(41,2)$ & $15(32,6)$ & $0,311^{1}$ & $43(46,2)$ & $19(28,4)$ & $0,022^{1}$ \\
\hline Visita acompanhamento grupos prioritários & $155(96,9)$ & $109(95,6)$ & $46(100)$ & $0,322^{2}$ & $90(96,8)$ & $65(97,0)$ & $1,000^{2}$ \\
\hline Organização de grupos terapêuticos & $21(13,1)$ & $18(15,8)$ & $3(6,5)$ & $0,116^{1}$ & $13(14)$ & $8(11,9)$ & $0,706^{1}$ \\
\hline Participação em reuniões da equipe & $26(16,3)$ & $16(14)$ & $10(21,7)$ & $0,232^{1}$ & $15(16,1)$ & $11(16,4)$ & $0,961^{1}$ \\
\hline Participação campanhas de vacinação humana & $47(29,4)$ & $33(28,9)$ & $14(30,4)$ & $0,852^{1}$ & $32(34,4)$ & $15(22,4)$ & $0,100^{1}$ \\
\hline $\begin{array}{l}\text { Visitas às famílias para prevenção de doenças em } \\
\text { geral }\end{array}$ & $56(35,0)$ & $44(38,6)$ & $12(26,1)$ & $0,133^{1}$ & $33(35,5)$ & $23(34,3)$ & $0,880^{1}$ \\
\hline Verificação de focos do Aedes aegypti & $28(17,5)$ & $24(21,1)$ & $4(8,7)$ & $0,063^{1}$ & $19(20,4)$ & $9(13,4)$ & $0,250^{1}$ \\
\hline Realizar palestras de educação em saúde & $44(27,5)$ & $33(28,9)$ & $11(23,9)$ & $0,519^{1}$ & $29(31,2)$ & $15(22,4)$ & $0,219^{1}$ \\
\hline $\begin{array}{l}\text { Cadastro e acompanhamento de outros programas } \\
\text { sociais }\end{array}$ & $15(9,4)$ & $12(10,5)$ & $3(6,5)$ & $0,557^{2}$ & $11(11,8)$ & $4(6,0)$ & $0,210^{1}$ \\
\hline \multicolumn{8}{|l|}{ Número de pessoas residentes na microárea } \\
\hline$<400$ pessoas & $41(25,6)$ & $8(7)$ & $33(71,7)$ & $<0,001^{2}$ & $23(24,7)$ & $18(26,9)$ & $1,000^{2}$ \\
\hline 400 a 750 pessoas & $85(53,1)$ & $74(64,9)$ & $11(23,9)$ & & $49(52,7)$ & $36(53,7)$ & \\
\hline 750 a 1.000 pessoas & $27(16,9)$ & $26(22,8)$ & $1(2,2)$ & & $16(17,2)$ & $11(16,4)$ & \\
\hline$>1.000$ pessoas & $6(3,8)$ & $6(5,3)$ & NA & & $4(4,3)$ & $2(3)$ & \\
\hline Não respondeu & $1(0,6)$ & $(\ldots)$ & $1(2,2)$ & & $1(1,1)$ & NA & \\
\hline Mantém o cadastro das famílias atualizado & $116(72,5)$ & $73(64,0)$ & $43(93,5)$ & $<0,001^{1}$ & $65(69,9)$ & $51(76,1)$ & $0,384^{1}$ \\
\hline $\begin{array}{l}\text { Visita todas as famílias de sua microárea } \\
\text { mensalmente }\end{array}$ & $118(73,8)$ & $17(14,9)$ & $25(54,3)$ & $<0,001^{1}$ & $23(24,7)$ & $19(28,4)$ & $0,607^{1}$ \\
\hline
\end{tabular}

${ }^{1}$ Teste Qui-Quadrado; ${ }^{2}$ Teste Exato de Fisher. 
Foi encontrada diferença estatisticamente significante entre as médias de idades dos ACS, de acordo com a zona onde trabalhavam, ou seja, os trabalhadores da ZR eram mais jovens (média: 37,8 anos; $\mathrm{DP}=9,3$ ) do que os da ZU (média: 42,8 anos; $\mathrm{DP}=9,2$ ) $(\mathrm{p}=0,004)$. Os ACS com menos de 10 anos de serviço têm maior escolaridade, e os com mais de 10 cursaram com mais frequência o CTACS (tabela 1). A proporção de ACS com ensino superior chegou a $35 \%$ entre os com menos de 10 anos de atuação.

Os ACS da ZR conseguem manter o cadastro das famílias atualizado e visitar todas as famílias de sua microárea mais frequentemente que os da ZU (tabela 2), estes últimos justificam que priorizam grupos vulneráveis.

A proporção de indivíduos dos grupos prioritários visitados mensalmente são: 91,9\% crianças < 2 anos; 91,3\% gestantes;
84,4\% diabéticos; $81,3 \%$ acamados e $66,3 \%$ hipertensos. A razão apontada mais frequentemente para não visitação de pessoas de grupos prioritários foi que estas não se encontravam em casa no horário de trabalho.

Quanto às práticas efetivadas durante as visitas domiciliares, chamou atenção a baixa proporção de aconselhamento às gestantes sobre amamentação, sinais de risco na gestação, cuidados com o recém-nascido, riscos do uso de tabaco e álcool, sendo que, para maioria dos desfechos, não houve diferenças entre a prática dos ACS por zona de trabalho ou tempo de serviço. Na tabela 3, estão descritas também a frequência de ações realizadas nas visitas aos diabéticos, e, neste caso, também se verificou baixa frequência de orientações para prevenir complicações, como problemas renais e oftalmológicos.

Tabela 3. Distribuição de ações domiciliares de saúde da gestante e do diabético segundo zona de atuação e tempo de serviço dos ACS, Fortaleza, Tauá, Eusébio e Cruz - Ceará, 2016

\begin{tabular}{|c|c|c|c|c|c|c|c|}
\hline & Total & Zona Urbana & Zona Rural & \multirow[t]{2}{*}{ Valor $\mathrm{p}$} & $<10$ anos & $>=10$ anos & \multirow[t]{2}{*}{ Valor $p$} \\
\hline & $N(\%)$ & $N(\%)$ & $\mathrm{N}(\%)$ & & $N(\%)$ & $N(\%)$ & \\
\hline \multicolumn{8}{|l|}{ Visita mensalmente todas as gestantes de sua área } \\
\hline Sim & $146(91,3)$ & $102(89,5)$ & $44(95,7)$ & $0,350^{2}$ & $85(91,4)$ & 61(91) & $0,575^{2}$ \\
\hline Não & $7(4,4)$ & $7(6,1)$ & NA & & $3(3,2)$ & $4(6)$ & \\
\hline Não há gestantes na minha microárea & $7(4,4)$ & $5(4,4)$ & $2(4,3)$ & & $5(5,4)$ & 2(3) & \\
\hline \multicolumn{8}{|l|}{ Ações durante a visita as gestantes } \\
\hline Verifica o cartão da gestante & $131(81,9)$ & $93(81,6)$ & $38(82,6)$ & $0,878^{1}$ & $77(82,8)$ & $54(80,6)$ & $0,722^{1}$ \\
\hline Certifica-se da realização da consulta pré-natal & $141(88,1)$ & $101(88,6)$ & $40(87)$ & $0,772^{1}$ & $78(83,9)$ & $63(94)$ & $0,050^{1}$ \\
\hline Orienta sobre alimentação na gestação & $75(46,9)$ & $54(47,4)$ & $21(45,7)$ & $0,844^{1}$ & $50(53,8)$ & $25(37,3)$ & $0,040^{1}$ \\
\hline Orienta sobre as vacinas & $128(80,0)$ & $93(81,6)$ & $35(76,1)$ & $0,432^{1}$ & $75(80,6)$ & $53(79,1)$ & $0,810^{1}$ \\
\hline Certifica-se da realização dos exames laboratoriais & $52(32,5)$ & $33(28,9)$ & $19(41,3)$ & $0,131^{1}$ & $30(32,3)$ & $22(32,8)$ & $0,939^{1}$ \\
\hline Orienta sobre amamentação & $56(35,0)$ & $36(31,6)$ & $20(43,5)$ & $0,153^{1}$ & $28(30,1)$ & $28(41,8)$ & $0,126^{1}$ \\
\hline Orienta cuidados com o recém-nascido & $27(16,9)$ & $20(17,5)$ & $7(15,2)$ & $0,722^{1}$ & $15(16,1)$ & $12(17,9)$ & $0,767^{1}$ \\
\hline $\begin{array}{l}\text { Orienta sobre riscos do uso de tabaco, álcool e } \\
\text { outras drogas }\end{array}$ & $10(6,3)$ & $8(7)$ & $2(4,3)$ & $0,725^{2}$ & $3(3,2)$ & $7(10,4)$ & $0,096^{2}$ \\
\hline $\begin{array}{l}\text { Orienta sobre prevenção de doenças sexualmente } \\
\text { transmissíveis }\end{array}$ & $6(3,8)$ & $5(4,4)$ & $1(2,2)$ & $0,674^{2}$ & $5(5,4)$ & $1(1,5)$ & $0,402^{2}$ \\
\hline $\begin{array}{l}\text { Pergunta se gestante apresentou algum mal estar } \\
\text { ou sinal de risco }\end{array}$ & $51(31,9)$ & $38(33,3)$ & $13(28,3)$ & $0,533^{1}$ & $33(35,5)$ & $18(26,9)$ & $0,248^{1}$ \\
\hline
\end{tabular}




\begin{tabular}{|c|c|c|c|c|c|c|c|}
\hline \multicolumn{8}{|l|}{ Visita todos os diabéticos de sua área mensalmente } \\
\hline $\operatorname{Sim}$ & $135(84,4)$ & $89(78,1)$ & $46(100)$ & $0,001^{1}$ & $76(81,7)$ & $59(88,1)$ & $0,276^{1}$ \\
\hline \multicolumn{8}{|c|}{ Ações que realiza quando visita uma pessoa com diabetes } \\
\hline Verifica uso de medicação & $151(94,4)$ & $108(94,7)$ & $43(93,5)$ & $0,717^{2}$ & $87(93,5)$ & $64(95,5)$ & $0,735^{2}$ \\
\hline Verifica prazo validade medicação & $48(30)$ & $39(34,2)$ & $9(19,6)$ & $0,067^{1}$ & $31(33,3)$ & $17(25,4)$ & $0,278^{1}$ \\
\hline Orienta alimentação e redução do açúcar & $126(78,8)$ & $83(72,8)$ & $43(93,5)$ & $0,004^{1}$ & $76(81,7)$ & $50(74,6)$ & $0,279^{1}$ \\
\hline Orienta necessidade de exercícios físicos & $79(49,4)$ & $52(45,6)$ & $27(58,7)$ & $0,134^{1}$ & $51(54,8)$ & $28(41,8)$ & $0,103^{1}$ \\
\hline $\begin{array}{l}\text { Pergunta sobre complicação (hipoglicemia, proble- } \\
\text { mas na visão, nos rins ou nos pés) }\end{array}$ & $36(22,5)$ & $20(17,5)$ & $16(34,8)$ & $0,018^{1}$ & $21(22,6)$ & $15(22,4)$ & $0,977^{1}$ \\
\hline Encaminha para serviço de urgência se necessário & $23(14,4)$ & $22(19,3)$ & $1(2,2)$ & $0,005^{1}$ & $12(12,9)$ & $11(16,4)$ & $0,532^{1}$ \\
\hline Verifica glicemia & $20(12,5)$ & $14(12,3)$ & $6(13)$ & $0,895^{1}$ & $13(14)$ & $7(10,4)$ & $0,505^{1}$ \\
\hline
\end{tabular}

\section{Resultados do estudo qualitativo}

As conversas produzidas nos GF e entrevistas revelaram a complexidade do trabalho dos ACS, e clarearam resultados do inquérito transversal. Os ACS, além de tentarem pôr em prática a programação estabelecida pelos protocolos do SUS e gestores de saúde, ao realizar seu trabalho, vivenciam como poucos a dureza da vida de comunidades empobrecidas. Além disso, ao morar na localidade onde trabalham, estão disponíveis para sua comunidade todos os dias da semana, inclusive em horários fora do expediente.

E assim, as próprias famílias elas precisam saber quais são realmente as atribuições do ACS, porque elas acham que a gente é o resolutor de tudo [...] do bolsa família ao cartão cidadão, a consulta [...] Assim a gente trabalha todo dia e... acorda cinco horas com uma ligação será que hoje tem médico? GF Tauazinho, Tauá-CE.

Seu escopo de práticas é de grande abrangência, englobando o ciclo de vida das famílias e os agravos de saúde prioritários, acompanhando o processo de vida, adoecimento e morte das pessoas.
Na verdade, nós somos encarregados de alimentar o consolidado do município né. Nós temos $N$ atividades, nós acompanhamos as crianças menores de dois anos, [...] puérperas [...], nós preenchemos o Esus, [...] e cadastramos as famílias [...] estamos fazendo um trabalho chamada a escala do Findrisk, onde [...], vemos o diâmetro da cintura e [...] um consenso de pessoas que poderão ter diabetes mellitus tipo II [...] visitas a acamados [...] com o subsídio a programas sociais no caso né que arremete a CRAS [Centro de Referência em Assistência Social]. GF Tauazinho, Tauá-CE.

As falas dos participantes evidenciam as competências de articulação intersetorial, busca ativa de casos e estratificação de indivíduos conforme critérios de risco para saúde, demonstrando a potencialidade dos ACS para a vigilância e promoção da saúde.

A gente tem aquele protocolo, chega na casa e primeiro buscar cartão de vacina [...] na conversa vai verificando se encontra algum fator de risco. GF I, Fortaleza-CE.

As arboviroses estão incluídas nas atividades dos ACS. "Nós também fazemos notificação de Chikungunya, dengue e zika vírus” GF Tauazinho, Tauá-CE. 
Ficou clara a diferença entre as realidades da ZU e ZR. Na primeira, revela-se a vida de moradores de bairros populares, escassos em serviços públicos, excluídos do consumo, sujeitos às mudanças frequentes de endereço, e submersos em um cotidiano violento relacionado com o tráfico de drogas. Nesses territórios, muitas vezes, os chefes do tráfico regulam a vida da comunidade e a visitação domiciliar dos ACS.

[...] a família se contrai né, por exemplo, tem uma criança dentro daquela casa que tem o traficante e não pode vim no posto se consultar e a esposa não pode porque eles são militares porque nessa saída [...] eles podem matar [...] essas crianças $[\ldots .$.$] vivem doente [...] muitas não vão à escola.$ GF II, Fortaleza-CE.

O tráfico de drogas está presente mesmo nos municípios de médio e pequeno porte, como é o caso de Tauá e Cruz. Apesar desse contexto ameaçador, os ACS procuram encontrar táticas para chegar às pessoas que necessitam de cuidados: "Eu consegui contato com a mulher do traficante, que tava gestante, por meio da vizinha dela" GF ZU, Cruz-CE.

$\mathrm{Na} Z \mathrm{R}$, o problema maior é o isolamento da população, dispersa em grandes extensões de semiárido, sem transportes públi$\cos$ ou particulares acessíveis. Tanto nos contextos de ZU como de ZR investigados, os ACS utilizam seu transporte pessoal e compram, com seu salário, o combustível para a visitação.

Os resultados de que apenas $14,9 \%$ dos ACS da ZU e 54,3\% da ZR visitarem todas as famílias mensalmente foi justificado pela adaptação da frequência e da duração das visitas de acordo com a gravidade da situação de cada família. Há famílias para as quais uma visita mensal é considerada desnecessária; há famílias com acamados ou puérperas que devem ser visitadas semanalmente; há situações, como a de pessoas com tuberculose não aderentes ao tratamento, em que a visita é diária. "Uma casa com um casal de adultos sem filhos, qual a necessidade de que seja visitada mensalmente?" GF ZU, Cruz-CE.

As exigências de produtividade feitas pelos gestores, equipe, e da própria comunidade, que demandam serviços do SUS, sobrecarregam os ACS.

Se... tivéssemos 25 horas pra trabalhar nós trabalharíamos... eu já sonhei eu fazendo um seminário... com todas as atribuições do ACS... que é pra população ficar a par... sonhei também pessoas que trabalham na hierarquia acima de nós passar uma semana com nós dentro da área. GF ZU, Tauá-CE.

Foi possível detectar o impacto de ações de educação permanente em saúde na prática dos ACS, em especial do Curso Técnico para ACS, do curso de extensão em Terapia Comunitária Integrativa (TCI) ${ }^{\mathbf{2 8 , 2 9}}$ e do curso Caminhos do Cuidado, relacionado com a prevenção do uso de drogas, como o crack.

Nós fizemos agora um curso né nosso curso técnico e ele foi assim de fundamental importância, porque ele fez um resgate na gente como ser humano. GF I, Fortaleza-CE.

Eu sou outra pessoa depois que fiz o curso de Roda Comunitária... eu organizo as reuniões quinzenalmente. Entrevista com ACS, Eusébio-CE.

A TCI é realizada por meio de reuniões com a comunidade, em que as pessoas presentes são convidadas a partilhar experiências e sabedorias de vida por meio do diálogo. Estudos têm evidenciado resultados positivos na abordagem ao sofrimento mental por meio dessa técnica ${ }^{29,30}$.

Condições mínimas para o trabalho no campo, como fardamento, equipamentos de proteção individual, máscaras, sapatos adequados, protetor ou roupas com fator de proteção solar, ajuda de custo para o combustível que utilizam, muitas vezes não são providos aos ACS. 


\section{Discussão}

Neste artigo, propusemo-nos a analisar o escopo de práticas dos ACS do Ceará relacionando-o à situação social e de saúde da população e à formação destes trabalhadores, bem como elementos potencializadores e limitantes desta prática. Os ACS identificaram como principal atividade a visitação domiciliar de grupos prioritários e o cadastramento de famílias no Siab e e-SUS AB. Estes achados são idênticos aos de um inquérito nacional sobre o perfil dos ACS, realizado em 2015, tendo participação de 1.526 ACS $^{31}$.

Como está descrito nos resultados, evidenciou-se a complexidade e abrangência do trabalho dos ACS, que inclui ações de vigilância à saúde, como busca ativa e notificação de doenças infecciosas, o encaminhamento de pessoas e famílias para unidade de saúde, garantindo a cobertura do pré-natal, imunizações, prevenção do câncer ginecológico, o controle da hipertensão e do diabetes, além do cuidado com idosos e pessoas acamadas ou domiciliadas, o que também foi verificado em outros estudos ${ }^{31}$. As atividades de preenchimento manual de instrumentos para alimentação de sistemas de informação diversos ocupa parte considerável do seu tempo. Estes são indicativos da importância da informatização desse processo ${ }^{32}$, com a entrada de dados durante as visitas por meio de tablets, o que liberaria tempo para a realização de outras atividades.

Se a ESF é a porta de entrada do SUS, os ACS são a porta de entrada da ESF, servindo de anteparo às insatisfações da população com o sistema, o que leva a intenso desgaste físico e emocional. Em estudo na cidade de São Paulo, observou-se que a prevalência de possível depressão maior em profissionais da ESF era 18\% mais elevada entre os $\mathrm{ACS}^{33}$. Entre as causas associadas, os autores apontaram maior tempo de trabalho na APS, grande pressão laboral, a falta de retroalimentação dos supervisores com respeito ao desempenho e o escasso apoio social dos colegas e supervisores ${ }^{33}$, situações também observadas nesta pesquisa.

Com o aumento da violência no Ceará, a visitação domiciliar dos ACS pode representar risco de vida, como reportam as falas sobre ameaças de traficantes, sobretudo na capital. Revelou-se também uma limitação de acesso de parte das mulheres e crianças aos serviços de saúde e educação, em consequência dos conflitos gerados por disputas de território entre facções criminosas. Em 2015, a violência urbana em Fortaleza foi identificada como causa de pedidos de demissão de trabalhadores em saúde em um bairro com população de baixa renda ${ }^{34}$. Neste estudo, levanta-se a hipótese de que a violência urbana, além da baixa cobertura da ESF, pode ter sido um dos elementos relacionados com as falhas de imunização de crianças com vacina antissarampo, que esteve associada ao surto de sarampo ocorrido em Fortaleza entre 2013 e $2015^{35}$.

Outra situação que causa estresse aos ACS é a grave deficiência no seu processo formativo, dado que, apesar da proposta de realização de um curso técnico de 1800 horas, $97,1 \%$ dos trabalhadores do estado ainda não o concluíram. O CTACS é composto por três etapas formativas. A primeira tem por objetivo desenvolver três competências nos participantes: promover ações para integração entre as equipes de saúde da família e a população; realizar, em conjunto com a equipe, atividades de planejamento e avaliação das ações de saúde; e desenvolver ações de promoção social e de proteção e desenvolvimento da cidadania. Na Etapa Formativa II, são desenvolvidas competências quanto à promoção da saúde de grupos específicos, como crianças, gestantes, pessoas com hipertensão, diabetes, tuberculose e hanseníase. As arboviroses e doenças veiculadas pela água são abordadas na Etapa Formativa III ${ }^{36}$.

A complexidade do processo de trabalho dos ACS e seu contato cotidiano com a população exigem um processo de educação

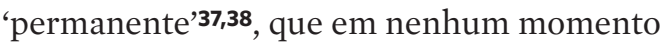


foi assumido integralmente pelas esferas de gestão do SUS $^{39}$. Os participantes expressaram claramente sua sensação de despreparo para realizar as ações de educação em saúde de forma a responder à superposição de agendas sanitárias.

Como fatores limitantes do escopo de práticas dos ACS, identificamos a deficiência no processo formativo, escasso apoio social de colegas e supervisores, sobrecarga de trabalho, a projeção de insatisfações da comunidade com o SUS sobre os ACS e a violência urbana. Como fatores facilitadores, identificamos a gestão participativa e investimentos em educação permanente, como o CTACS, o Curso em Terapia Comunitária e o Caminhos do Cuidado, relacionado com a prevenção do uso de drogas e com o cuidado com pessoas dependentes.

\section{Considerações finais}

O presente trabalho evidenciou a complexidade do trabalho do ACS, que inclui ações de promoção e vigilância à saúde, como pré-natal, imunizações, hipertensão, diabetes, cuidado com idosos, entre outros. Como limitantes das práticas, identificou-se: deficiência da formação técnica, suporte reduzido no trabalho e violência, que tem afetado sua atuação profissional de forma incontesta. Contudo, potencializadores do trabalho do ACS também foram identificados, como a educação permanente e a gestão participativa, encontrada em algumas realidades avaliadas. O escopo de práticas dos ACS é complexo e abrangente, incluindo a articulação de políticas públicas no território, o que se constitui em uma potencialidade para promoção da saúde de comunidades vulneráveis.

A continuidade do trabalho dos ACS está sendo questionada pelos gestores do SUS por meio da PNAB 2017 e do Profags, este último pode ser inclusive interpretado como uma tentativa de desprofissionalização desses trabalhadores da saúde. Os autores afirmam que se no trabalho dos ACS verifica-se um acúmulo de problemas que refletem a própria situação social e de saúde de suas comunidades, e os limites da ESF e do SUS, neste encontra-se também um potencial importante de contribuição para superação destes problemas, dado o seu papel de articulador de diversas políticas públicas, como saúde, educação e assistência social em comunidades vulneráveis. Cabe ao Estado brasileiro cumprir seu papel de garantir a formação necessária e o suporte adequado a estes trabalhadores.

\section{Colaboradores}

Barreto ICHC contribuiu substancialmente para a concepção do manuscrito, para o projeto de pesquisa, contribuiu para aquisição, análise e para interpretação dos dados para o trabalho, realizou a redação do trabalho, contando com a revisão crítica dos coautores, aprovou a versão final a ser publicada e manifesta concordância em assumir responsabilidade por todos os aspectos do trabalho, assegurando que as perguntas relacionadas com precisão ou integridade de qualquer parte do estudo serão apropriadamente investigadas e resolvidas. Pessoa VM contribuiu substancialmente para a elaboração do projeto de pesquisa, contribuiu para análise e para interpretação dos dados, realizou revisão crítica e aprovou a versão final a ser publicada, manifestando concordância em assumir responsabilidade por todos os aspectos do trabalho, assegurando que as perguntas relacionadas com precisão ou integridade de qualquer parte do estudo serão apropriadamente investigadas e resolvidas. Sousa MFA contribuiu substancialmente para a elaboração do projeto de pesquisa, contribuiu para aquisição, análise e para interpretação dos dados, realizou revisão crítica e aprovou a versão 
final a ser publicada, manifestando concordância em assumir responsabilidade por todos os aspectos do trabalho, assegurando que as perguntas relacionadas com precisão ou integridade de qualquer parte do estudo serão apropriadamente investigadas e resolvidas. Nuto SAS contribuiu substancialmente para a elaboração do projeto de pesquisa, contribuiu para aquisição, análise e para interpretação dos dados, realizou revisão crítica e aprovou a versão final a ser publicada, manifestando concordância em assumir responsabilidade por todos os aspectos do trabalho, assegurando que as perguntas relacionadas com precisão ou integridade de qualquer parte do estudo serão apropriadamente investigadas e resolvidas. Freitas RWJF contribuiu substancialmente para a elaboração do projeto de pesquisa, contribuiu para análise e para interpretação dos dados, realizou revisão crítica e aprovou a versão final a ser publicada, manifestando concordância em assumir responsabilidade por todos os aspectos do trabalho, assegurando que as perguntas relacionadas com precisão ou integridade de qualquer parte do estudo serão apropriadamente investigadas e resolvidas. Ribeiro KG contribuiu substancialmente para a redação do manuscrito, contribuiu para análise e para interpretação dos dados, realizou revisão crítica e aprovou a versão final a ser publicada, manifestando concordância em assumir responsabilidade por todos os aspectos do trabalho, assegurando que as perguntas relacionadas com precisão ou integridade de qualquer parte do estudo serão apropriadamente investigadas e resolvidas. Vieira-Meyer APGF contribuiu para a interpretação dos dados, realizou revisão crítica e aprovou a versão final a ser publicada, manifestando concordância em assumir responsabilidade por todos os aspectos do trabalho, assegurando que as perguntas relacionadas com precisão ou integridade de qualquer parte do estudo serão apropriadamente investigadas e resolvidas. Andrade LOM contribuiu para análise e para interpretação dos dados, realizou revisão crítica e aprovou a versão final a ser publicada, manifestando concordância em assumir responsabilidade por todos os aspectos do trabalho, assegurando que as perguntas relacionadas com precisão ou integridade de qualquer parte do estudo serão apropriadamente investigadas e resolvidas.

\section{Agradecimentos}

À Nísia Trindade Lima e a toda a equipe de gestão da Fiocruz, por possibilitarem a realização desta pesquisa. À Isabela Soares Santos e à Roberta Goldstein, coordenadoras da Rede de Pesquisas em Políticas Públicas e Sistemas de Saúde da Fiocruz, pelo suporte à pesquisa. Ao Dr. Antônio Carlile Holanda Lavor pelas contribuições na elaboração dos instrumentos do estudo e análise dos dados. Ao Edgar Sampaio, pelas análises estatísticas, e à Rubia Andrade, pela revisão das referências bibliográficas. 


\section{Referências}

1. Organização Mundial de Saúde; Fundo das Nações Unidas para a Infância. Alma Ata 1978: Cuidados Primários de Saúde. Brasília, DF: Unicef; 1979 [acesso em 2017 nov 24]. Disponível em: http://apps.who. int/iris/bitstream/10665/39228/5/9241800011_ por.pdf.

2. Svitone EC, Garfield R, Vasconcelos MI, et al. Primary health care lessons from the Northeast of Brazil: the Agentes de Saúde Program. Rev Panam Salud Pública. 2000; 7(5):293-301.

3. Medeiros CN, Pinho Neto VR. Análise espacial da extrema pobreza no Estado do Ceará. Ceará: Ipece; 2010 [acesso em 2017 dez 2]. Disponível em: http:// www.ipece.ce.gov.br/encontro_economia/vii_encontro/artigos/ANALISE_ESPACIAL_DA_EXTREMA_POBREZA_NO_ESTADO_DO_CEARA. pdf.

4. Brasil. Ministério da Saúde. Datasus - Tabnet - Informações de Saúde - Demográficas e Socioeconômicas - Índice de Gini da renda domiciliar per capita segundo Estado. Brasília, DF: MS; 2010.

5. Ferreira VSC, Andrade CS, Franco TB, et al. Processo de trabalho do agente comunitário de saúde e a reestruturação produtiva. Cad Saúde Pública. 2009; 5(4):898-906.

6. Poz M, Viana A. A Reforma no Sistema de Saúde no Brasil e o Programa de Saúde da Família. Physis. 1998; 8(2):11-48.

7. Brasil. Ministério da Saúde. Secretaria de Atenção à Saúde. Memórias da saúde da família no Brasil [internet]. Brasília, DF: Ministério da Saúde; 2010 [acesso em 2017 dez 16]. Disponível em: http://bvsms.saude.gov.br/bvs/publicacoes/memorias_saude_familia_brasil.pdf.

8. Brasil. Presidência da República. Decreto Federal 7.508. Regulamenta a Lei no 8.080, de 19 de setembro de 1990. Dispõe sobre a organização do Sistema Único de Saúde - SUS, o planejamento da saúde, a assistência à saúde e a articulação interfederativa, e dá outras providências. Diário Oficial da União. 19 Set 1990 [acesso em 2017 dez 2]. Disponível em: http://www.planalto.gov.br/ccivil_03/_ato20112014/2011/decreto/d7508.htm.

9. Brasil. Presidência da República. Lei $\mathrm{n}^{0}$ 11.350, de 11 de outubro de 2006. Regulamenta o 95 o do art. 198 da Constituição. Dispõe sobre o aproveitamento de pessoal amparado pelo parágrafo único do art. $2^{\circ}$ da Emenda Constitucional ${ }^{\circ} 51$, de 14 de fevereiro de 2006, e dá outras providências. Diário Oficial da União. 14 Fev 2006 [acesso em 2017 jan 22]. Disponível em: http://www.planalto.gov.br/ccivil_03/ato2004-2006/2006/lei/111350.htm.

10. Brasil. Ministério da Saúde. Departamento de Atenção Básica. Histórico da cobertura da estratégia saúde da família [internet]. Brasília, DF: MS; 2017 [acesso em 2017 nov 15]. Disponível em: http:// dab.saude.gov.br/portaldab/historico_cobertura sf.php.

11. Instituto Brasileiro de Geografia e Estatística. Projeção da população do Brasil e das unidades da federação [internet]. Rio de Janeiro: IBGE; 2017 [acesso em 2017 nov 15]. Disponível em: http://www.ibge. gov.br/apps/populacao/projecao.

12. Pontes RJS, Ramos Júnior AN, Kerr LRS, et al. Transição demográfica e epidemiológica. In: Medronho RA. Epidemiologia. Rio de Janeiro: Atheneu; 2009. p. 49-68.

13. Barreto ML, Teixeira MG, Bastos FI, et al. Sucessos e fracassos no controle de doenças infecciosas no Brasil: o contexto social e ambiental, políticas, intervenções e necessidades de pesquisa. The Lancet [internet]. 2011 [acesso em $2017 \mathrm{dez}$ 17]; 377:18771889. Disponível em: http://www.thelancet.com/ $\mathrm{pb} /$ assets/raw/Lancet/pdfs/brazil/brazilpor3.pdf.

14. Ali S, Gugliemini O, Harber S, et al. Environmental and Social Change Drive the Explosive Emergence of Zika Virus in the Americas. PLoS Negl Trop Dis 
[internet]. 2017; 11(2):1-16.

15. Schmidt MI, Duncan BB, Silva GA, et al. Chronic non-communicable diseases in Brazil: burden and current challenges. The Lancet [internet]. 2011 [acesso em 2017 dez 16]; 377(9781):1949-1961. Disponível em: http://www.thelancet.com/journals/ lancet/article/PIIS0140-6736(11)60135-9/abstract.

16. Yunes J. Mortality from Violent Causes in the Américas. Bulletin of PAHO. 1993; 114(4):302-316.

17. Paiva T. Homicídios ultrapassam AVC nas causas de morte em Fortaleza. O Povo [internet]. 2017 nov 29 [acesso em 2017 dez 16]. Disponível em: https:// www.opovo.com.br/jornal/cotidiano/2017/11/homicidios-ultrapassam-avc-nas-causas-de-morte-em-fortaleza.html.

18. Lavor AC, Lavor MC, Lavor IC. Agente Comunitário de Saúde: um novo profissional para novas necessidades da saúde. SANARE [internet]. 2004 [acesso em 2017 dez 16]; 5(1):121-128. Disponível em: https://sanare.emnuvens.com.br/sanare/article/download/130/122

19. Ursua RA, Aguilar DE, Wyatt LC, et al. A Community Health Worker Intervention to Improve Management of Hypertension among Filipino Americans in New York and New Jersey: A Pilot Study. Ethn Dis. 2014; 24(1):67-76.

20. Herce ME, Chapman JA, Castro A, et al. A role for community health promoters in tuberculosis control in the state of Chiapas, Mexico. J Community Health. 2010; 35(2):182-189.

21. Brasil. Ministério da Saúde. Portaria no 2.436, de 21 de setembro de 2017. Aprova a Política Nacional de Atenção Básica, estabelecendo a revisão de diretrizes para a organização da Atenção Básica, no âmbito do Sistema Único de Saúde (SUS). Diário Oficial da União. 21 Set 2017.

22. Brasil. Ministério da Saúde. Portaria GM n ${ }^{\circ} 83,10$ de janeiro de 2018. Institui o Programa de Formação Técnica para Agentes de Saúde - PROFAGS, para oferta de curso de formação técnica em enfermagem para Agentes Comunitários de Saúde (ACS) e Agentes de Combates às Endemias (ACE) no âmbito do SUS, para o biênio de 2018-2019. Diário Oficial da União. 10 Jan 2018.

23. Pontes ALM, Bornstein VJ, Giugliani C. O Agente Comunitário de Saúde em Angola: desafios para sua atuação e para a formação profissional em saúde. Trab Educ Saúde. 2011; 9(3):521-533.

24. Creswell JW. Projeto de Pesquisa: métodos qualitativo, quantitativo e misto. Porto Alegre: Artmed; 2010.

25. Somekh CL, Lewin C. Teoria e Métodos de Pesquisa Social. Rio de Janeiro: Vozes; 2015.

26. Instituto de Planejamento e Gestão do Ceará [internet]. Fortaleza: IPECE; 2017 [acesso em $2017 \mathrm{dez}$ 17]. Disponível em: http://www2.ipece.ce.gov.br/ atlas/.

27. Bardin L. Análise de Conteúdo. Rio de Janeiro: Edições 70; 2011.

28. Brasil. Ministério da Saúde. O SUS e a Terapia Comunitária. Andrade LOM, Barreto ICHC, Barreto AP, et al., organizadores. Brasília, DF: 2009 [acesso em 2107 dez 17]. Disponível em: https://issuu.com/ abratecomterapiacomunitaria/docs/livrossustc.

29. Ianine AR, Lucineide AVB, Lucídia MT, et al. A terapia comunitária como um novo instrumento de cuidado para saúde mental do idoso instrumento de cuidado para saúde mental do idoso. Rev Bras Enferm [internet]. 2009 [acesso em $2017 \mathrm{dez}$ 17]; 62(5):687-694. Disponível em: http://www.scielo. br/pdf/reben/v62n5/06.

30. Ferreira Filha MO, Dias MD, Andrade FB, et al. A terapia comunitária como estratégia de promoção à saúde mental: o caminho para o empoderamento. Rev Eletron Enf [internet]. 2009; 11(4):964-970 [acesso em 2017 dez 17]. Disponível em: http://www. revistas.ufg.br/fen/article/download/33252/17588. 
31. Nunes AC, Silva IV. Avaliação do Perfil dos Agentes Comunitários de Saúde no Processo de Consolidação da Atenção Primária à Saúde no Brasil. Salvador: UFBA; 2016.

32. Brasil. Ministério da Saúde. E-SUS Atenção Básica: Manual do Aplicativo ACS. Brasília, DF: MS; 2015 [acesso em 2017 dez 17]. Disponível em: http://189.28.128.100/dab/docs/portaldab/documentos/Manual_esus_ab_territorio_PRELIMINAR.pdf.

33. Silva ATC, Lopes CS, Susser E, et al. Work-Related Depression in Primary Care Teams in Brazil. Am J Public Health [internet]. 2016 [acesso em $2017 \mathrm{dez}$ 17]; 106(11):1990-7. Disponível em: https://www. ncbi.nlm.nih.gov/pubmed/27631749.

34. Ribeiro KG. Os Determinantes Sociais em Saúde no Grande Bom Jardim - Fortaleza (CE) [tese]. Fortaleza: UFC; 2015 [acesso em 2017 dez 17]. 360 p. Disponível em: http://www.repositorio.ufc.br/bitstream/riufc/11980/1/2015_tese_kgribeiro.pdf.

35. Lemos DRQ, Franco AR, Roriz MLFS, et al. Measles epidemic in Brazil in the post-elimination period: Coordinated response and containment strategies. Vaccine [internet]. 2017 [acesso em 2017 dez 17]; 35(13):1721-1728. Disponível em: http:// www.sciencedirect.com/science/article/pii/ S0264410X17302177\#f0015.
36. Ceará. Escola de Saúde Pública do Ceará. Diretoria de Educação Profissional em Saúde. Relatório Técnico do Curso de Agente Comunitário de Saúde: Etapa Formativa I. Fortaleza: [s.n]; [s. d]. 24 p.

37. Haddad J, Roschke M, Davini C. Educación permanente en salud. Washington: OPAS; 1995.

38. Brasil. Ministério da Saúde. Portaria no 1.996, de 20 de agosto de 2007. Dispõe sobre as diretrizes para a implementação da Política Nacional de Educação Permanente em Saúde. Diário Oficial da União. 20 Ago 2007. [acesso em 2018 out 14]. Disponível em: http://bvsms.saude.gov.br/bvs/saudelegis/ gm/2007/prt1996_20_08_2007.html.

39. Simas PRP, Pinto ICM. Trabalho em saúde: retrato dos agentes comunitários de saúde da região Nordeste do Brasil. Ciênc Saúde Colet. [internet]. 2017 jun [acesso em 2018 maio 30]; 22(6):1865-1876. Disponível em: http://www.scielo.br/scielo.php?script=sci arttext\&pid=S1413-81232017002601865\&lng=en .

Recebido em 30/05/2017

Aprovado em 28/08/2018

Conflito de interesses: inexistente

Suporte financeiro: não houve 